\title{
AN APPROXIMATE ANALYTICAL TECHNIQUE FOR SOLVING SECOND ORDER STRONGLY NONLINEAR GENERALIZED DUFFING EQUATION WITH SMALL DAMPING
}

\author{
M. ALHAZ UDDIN*, M. WALI ULLAH ${ }^{1}$ AND REHANA SULTANA BIPASHA ${ }^{2}$ \\ Department of Mathematics, Khulna University of Engineering and Technology, \\ Khulna-9203, Bangladesh
}

\begin{abstract}
In this paper, He's homotopy perturbation method has been extended for obtaining the analytical approximate solution of second order strongly nonlinear generalized duffing oscillators with damping based on the extended form of the Krylov-Bogoliubov-Mitropolskii (KBM) method. Accuracy and validity of the solutions obtained by the presented method are compared with the corresponding numerical solutions obtained by the well-known fourth order Rangue-Kutta method. The method has been illustrated by examples.
\end{abstract}

Key words: Homotopy perturbation, KBM methods, Second order strongly nonlinear generalized Duffing oscillator

\section{INTRODUCTION}

The study of nonlinear problems in all areas of applied mathematics, physics, engineering, medical science, economics and other disciplines is of crucial importance, since most of the phenomena in the real world are essentially nonlinear and described by the nonlinear differential systems with small damping. It is difficult to handle nonlinear problems and in general, it is often very difficult to get an approximate analytical solution for strongly nonlinear generalized differential systems with damping than a numerical one. The most well-known common methods for constructing the approximate analytical solutions to the nonlinear oscillators are the perturbation techniques. Among of these techniques are the Krylov-Bogoliubov-Mitropolskii (KBM) (Krylov 1947, Bogoliubov and Mitropolskii 1961, Mitropolskii 1964) method, the Lindstedt-Poincare (LP) method (Nayfeh 1981, Murdock 1991), and the method of multiple time scales (Nayfeh 1981). Perturbation methods are based on an assumption that small parameters must exist in the equations, which is too strict to find wide application of the classical perturbation techniques. It determines not only the accuracy of the perturbation approximations, but also the validity of the perturbation methods itself. However, in science and engineering,

\footnotetext{
* Corresponding author: <alhazuddin@yahoo.com>.

${ }^{1}$ Mathematics, Department of Business Administration, Northern University Bangladesh.

${ }^{2}$ Mathematics, Department of Business Administration, Asian University of Bangladesh, Dhaka1230, Bangladesh.
} 
there exist many nonlinear oscillatory problems which do not contain any small parameter, especially those appear in nature with strong nonlinearities. Therefore, many new techniques have been proposed to eliminate the "small parameter" assumption. Among them, the homotopy perturbation method (HPM) is a popular one. Recently $\mathrm{He}$ (1998) obtained the approximate solution of nonlinear differential equation with convolution product nonlinearities. He (1999) also developed some new approaches to Duffing equation with strongly and high order nonlinearity and presented a new interpretation of homotopy perturbation method (He 2006). Belendez et al. (2007) presented the application of He's homotopy perturbation method to Duffing harmonic oscillator. Lim et al. (2003) also presented a new analytical approach to the Duffingharmonic oscillator. Alam et al. (2006) presented a general Struble's technique for solving an $n$th order weakly nonlinear differential systems with damping. Azad et al. (2006) presented KBM asymptotic method for over-damped processes in biological and biochemical systems. Uddin et al. (2011) developed an approximate technique for solving strongly cubic nonlinear differential systems with damping effects. Uddin and Sattar (2011) also presented an approximate technique to Duffing equation with small damping and slowly varying coefficients. Uddin (2011) applied He's homotopy perturbation method to Duffing equation with small damping and high order strongly nonlinearities. Recently Uddin et al. (2012) developed an approximate analytical technique for solving certain type of fourth order strongly nonlinear oscillatory differential systems with small damping and cubic nonlinearity. Younesian et al. (2010) described frequency analysis of strongly nonlinear generalized Duffing oscillators using He's frequency- amplitude formulation and He's energy balance method. In present study it has been seen that the most of the authors (He 1998, 1999, 2006, Belendez et al. 2007, Younesian et al. 2010) studied nonlinear differential systems without considering any damping effects. Most of the physical and engineering problems occur in nature as nonlinear differential systems with small damping. The aim of this work was to develop an analytical approximate technique by coupling the homotopy perturbation and the extended form of the KBM methods for solving the second order strongly nonlinear generalized Duffing equation with small damping.

\section{MATERIALS AND METHOD}

The authors were interested to consider the strongly nonlinear generalized Duffing oscillator with small damping modeling in the following form

$$
\ddot{x}+2 k \dot{x}+v^{2} x=-\varepsilon\left(\alpha_{3} f_{3}(x, \dot{x})+\alpha_{5} f_{5}(x, \dot{x})+\alpha_{7} f_{7}(x, \dot{x})+\cdots+\alpha_{n} f_{n}(x, \dot{x})\right),
$$

under the initial conditions

$$
x(0)=b_{0}, \quad \dot{x}(0)=0,
$$


where over dots denote differentiations with respect to time $t, v$ is a constant, $\varepsilon$ is a parameter not necessarily small, $2 k$ is the linear damping coefficient, $\alpha_{i}$ are constants $b_{0}$ is an initial amplitude, and $f_{i}(x, \dot{x}), i=3,5,7 \cdots n$ are given nonlinear functions which satisfies the following condition

$$
f_{i}(-x,-\dot{x})=-f_{i}(x, \dot{x}) \text {. }
$$

To solve the Eq. (1), the present workers assumed the following transformation

$$
x=y(t) e^{-k t} .
$$

Now differentiating Eq. (4) twice with respect to time $t$ and substituting $\ddot{x}, \dot{x}$ together with $x$ into Eq. (1), we obtain

$$
\ddot{y}+\left(v^{2}-k^{2}\right) y=-\varepsilon e^{-k t} \sum_{i=1}^{n} \alpha_{i} f_{i}\left(y e^{-k t},(\dot{y}-k y) e^{-k t}\right) .
$$

According to the homotopy perturbation method, Eq. (5) can be re-written as

$$
\ddot{y}+\omega^{2} y=\lambda y-\varepsilon e^{-k t} \sum_{i=1}^{n} \alpha_{i} f_{i}\left(y e^{-k t},(\dot{y}-k y) e^{-k t}\right),
$$

where

$$
\omega^{2}=v^{2}-k^{2}+\lambda
$$

Herein $\omega$ is a constant for undamped nonlinear oscillators and known as the frequency of the nonlinear systems and $\lambda$ is an unknown function which can be determined by eliminating the secular terms. But for the damped nonlinear differential systems $\omega$ is a time dependent function and it varies slowly with time $t$. To handle this situation, the authors used the extended form of the KBM (Krylov 1947, Bogoliubov and Mitropolskii 1961, Mitropolskii 1964) method. According to this method. They chose the solution of Eq. (6) in the following form

$$
y=b \cos \psi,
$$

where $b$ and $\psi$ vary slowly with time $t$. In literature, $b$ and $\psi$ are known as the amplitude and phase variables respectively and they keep an important role to nonlinear physical systems. The amplitude $b$ and phase $\psi$ satisfy the following first order ordinary differential equations

$$
\begin{aligned}
& \dot{b}=\varepsilon B_{1}(b, \tau)+\varepsilon^{2} B_{2}(b, \tau)+\cdots, \\
& \dot{\psi}=\omega(\tau)+\varepsilon C_{1}(b, \tau)+\varepsilon^{2} C_{2}(b, \tau)+\cdots,
\end{aligned}
$$

where $\varepsilon$ is a small positive parameter and $\tau=\varepsilon t$ is the slowly varying time. Now differentiating Eq. (8) twice with respect to time $t$, utilizing the relations Eq. (9) and substituting $\ddot{y}, y$ into Eq. (6) and then equating the coefficients of $\sin \varphi$ and $\cos \varphi$, the authors obtained 


$$
B_{1}=-\omega^{\prime} b /(2 \omega), C_{1}=0,
$$

where prime denotes differentiation with respect to slowly varying time $\tau$. Now putting Eq. (8) into Eq. (4) and Eq. (10) into Eq. (9), the authors obtained the following equations

$$
\begin{aligned}
& x=b e^{-k t} \cos \psi, \\
& \dot{b}=-k \omega^{\prime} b /(2 \omega), \\
& \dot{\psi}=\omega(\tau) .
\end{aligned}
$$

Thus, the first order analytical approximate solution of Eq. (1) is given by the presented coupling technique by Eq. (11) with the help of Eqs (7) and (12). Usually the integration of Eq. (12) is computed by well-known techniques of calculus (Nayfeh 1981, Murdock 1991), but often these are calculated by a numerical procedure (Alam et al. 2006, Arya and Bojadziev 1981, Bojadziev 1983, Uddin et al. 2011).

Examples: 3.1 To apply the above procedure for the practical problems, one may consider the strongly nonlinear generalized Duffing equation with a linear damping effects for $n=3,5$ (He 1998, 1999, 2006, Younesian et al. 2010, Uddin et al. 2012) in the following form

$$
\ddot{x}+2 k \dot{x}+v^{2} x=-\varepsilon\left(\alpha_{3} x^{3}+\alpha_{5} x^{5}\right),
$$

where $f_{3}(x, \dot{x})=x^{3}, f_{5}(x, \dot{x})=x^{5}$. Now using the transformation Eq. (4) into Eq. (13) and then simplifying them, one obtains

$$
\ddot{y}+\left(v^{2}-k^{2}\right) y=-\varepsilon\left(\alpha_{3} y^{3} e^{-2 k t}+\alpha_{5} y^{5} e^{-4 k t}\right) .
$$

According to the homotopy perturbation technique, Eq. (14) can be written as

$$
\ddot{y}+\omega^{2} y=\lambda y-\varepsilon\left(\alpha_{3} y^{3} e^{-2 k t}+\alpha_{5} y^{5} e^{-4 k t}\right),
$$

where $\omega$ is given by Eq. (7). According to the extended form of the KBM (Krylov 1947, Bogoliubov and Mitropolskii 1961, Mitropolskii 1964) method, the solution of Eq. (15) is obtained from Eq. (8).

The requirement of no secular terms in particular solution of Eq. (15) implies that the coefficients of the $\cos \varphi$ terms are zero. Setting these terms to zero, one may obtain

$$
\lambda b-\frac{3 \varepsilon \alpha_{3} b^{3} e^{-2 k t}}{4}-\frac{5 \varepsilon \alpha_{5} b^{5} e^{-4 k t}}{16}=0,
$$

which leads to

$$
\lambda=\frac{3 \varepsilon \alpha_{3} b^{2} e^{-2 k t}}{4}+\frac{5 \varepsilon \alpha_{5} b^{4} e^{-4 k t}}{16} .
$$


Putting the value of $\lambda$ from Eq. (17) into Eq. (6), then it leads to

$$
\omega^{2}=v^{2}-k^{2}+\frac{3 \varepsilon \alpha_{3} b^{2} e^{-2 k t}}{4}+\frac{5 \varepsilon \alpha_{5} b^{4} e^{-4 k t}}{16} .
$$

From Eq. (18) it is clear that, the frequency of the damped nonlinear differential systems depends on both amplitude $b$ and time $t$. When $t \rightarrow 0$ then Eq. (18) yields

$$
\omega_{0}=\omega(0)=\sqrt{v^{2}-k^{2}+\frac{3 \varepsilon \alpha_{3} b_{0}^{2}}{4}+\frac{5 \varepsilon \alpha_{5} b_{0}^{4}}{16}} .
$$

By integrating Eq. (12), one can get

$$
b=b_{0} \sqrt{\frac{\omega_{0}}{\omega}}, \quad \varphi=\varphi_{0}+\int_{0}^{t} \omega(\tau) d t,
$$

where $b_{0}$ and $\varphi_{0}$ are constants of integration and is known as the initial amplitude and phase of the systems. Now putting Eq. (20) into Eq. (18), one may obtain a biquadratic algebraic equation in $\omega$ in the following form

$$
\omega^{4}+p \omega^{2}+q \omega+r=0,
$$

where

$$
p=k^{2}-v^{2}, q=-\frac{3 \varepsilon \alpha_{3} \omega_{0} b_{0}^{2} e^{-2 k t}}{4}, r=-\frac{5 \varepsilon \alpha_{5} \omega_{0}{ }^{2} b_{0}^{4} e^{-4 k t}}{16} .
$$

The solution of Eq. (21) is computed by using the well-known Newton-Raphson method. Thus, the first order analytical approximate solution of Eq (13) is given by

$$
\begin{aligned}
& x=b e^{-k t} \cos \psi, \\
& b=b_{0} \sqrt{\frac{\omega_{0}}{\omega}}, \quad \varphi=\varphi_{0}+\int_{0}^{t} \omega(\tau) d t
\end{aligned}
$$

where $\omega_{0}$ is obtained by Eq. (19), $\omega$ is calculated from Eq. (21), $b$ and $\psi$ are carried out by Eq. (24).

3.2 As a second example, one may consider the strongly nonlinear generalized Duffing oscillator with a linear damping effects (He 1998, 1999, 2006, Younesian et al. 2010, Uddin et al. 2012) modeling in the following form

$$
\ddot{x}+2 k \dot{x}+v^{2} x=-\varepsilon\left(\alpha_{3} x^{3}+\alpha_{5} x^{5}+\alpha_{7} x^{7}\right) .
$$

Now by using the transformation Eq. (4) into Eq. (25) and then simplifying them, one may obtain

$$
\ddot{y}+\left(v^{2}-k^{2}\right) y=-\varepsilon\left(\alpha_{3} y^{3} e^{-2 k t}+\alpha_{5} y^{5} e^{-4 k t}+\alpha_{7} y^{7} e^{-6 k t}\right) .
$$


According to the homotopy perturbation method, Eq. (26) yields

$$
\ddot{y}+\omega^{2} y=\lambda y-\varepsilon\left(\alpha_{3} y^{3} e^{-2 k t}+\alpha_{5} y^{5} e^{-4 k t}+\alpha_{7} y^{7} e^{-6 k t}\right),
$$

where $\omega$ is obtained by Eq. (7). According to the extended form of the KBM (Krylov 1947, Bogoliubov and Mitropolskii 1961, Mitropolskii 1964) method, the solution of Eq. (27) is performed by Eq. (8).

From the trigonometric identity, one can obtain

$$
\cos ^{7} \varphi=(\cos 7 \varphi+7 \cos 5 \varphi+21 \cos 3 \varphi+35 \cos \varphi) / 64 .
$$

The requirement of no secular terms in particular solution of Eq. (27) implies that the coefficients of the $\cos \varphi$ terms are zero. Setting these terms to zero, one may obtain

$$
\lambda b-\frac{3 \varepsilon \alpha_{3} b^{3} e^{-2 k t}}{4}-\frac{5 \varepsilon \alpha_{5} b^{5} e^{-4 k t}}{16}-\frac{35 \varepsilon \alpha_{7} b^{7} e^{-6 k t}}{64}=0,
$$

which leads to

$$
\lambda=\frac{3 \varepsilon \alpha_{3} b^{2} e^{-2 k t}}{4}+\frac{5 \varepsilon \alpha_{5} b^{4} e^{-4 k t}}{16}+\frac{35 \varepsilon \alpha_{7} b^{6} e^{-6 k t}}{64} .
$$

Putting the value of $\lambda$ from Eq. (30) into Eq. (7), yields

$$
\omega^{2}=v^{2}-k^{2}+\frac{3 \varepsilon \alpha_{3} b^{2} e^{-2 k t}}{4}+\frac{5 \varepsilon \alpha_{5} b^{4} e^{-4 k t}}{16}+\frac{35 \varepsilon \alpha_{7} b^{6} e^{-6 k t}}{64} .
$$

From Eq. (31), one may obtain (as $t \rightarrow 0$ )

$$
\omega_{0}=\omega(0)=\sqrt{v^{2}-k^{2}+\frac{3 \varepsilon \alpha_{3} b_{0}^{2}}{4}+\frac{5 \varepsilon \alpha_{5} b_{0}^{4}}{16}+\frac{35 \varepsilon \alpha_{7} b_{0}^{6}}{64}} .
$$

By integrating Eq. (12) and using it into Eq. (31), one can obtain a fifth degree algebraic equation in $\omega$ in the following form

$$
\omega^{5}+p \omega^{3}+q \omega^{2}+r \omega+s=0,
$$

where

$$
\begin{aligned}
& p=k^{2}-v^{2}, \quad q=-\frac{3 \varepsilon \alpha_{3} \omega_{0} b_{0}^{2} e^{-2 k t}}{4} \\
& r=-\frac{5 \varepsilon \alpha_{5} \omega_{0}{ }^{2} b_{0}{ }^{4} e^{-4 k t}}{16}, s=-\frac{35 \varepsilon \alpha_{7} \omega_{0}{ }^{3} b_{0}{ }^{6} e^{-6 k t}}{64} .
\end{aligned}
$$

The solution of Eq. (33) is obtained by using the well-known Newton-Raphson method.

Thus, the first order analytical approximate solution of Eq (25) is given by

$$
x=b e^{-k t} \cos \psi,
$$




$$
b=b_{0} \sqrt{\frac{\omega_{0}}{\omega}}, \quad \varphi=\varphi_{0}+\int_{0}^{t} \omega(\tau) d t,
$$

where $\omega_{0}$ is obtained by Eq. (32), $\omega$ is calculated from Eq. (33), $b$ and $\psi$ are given by Eq. (36).

\section{RESULTS AND DISCUSSION}

In this paper, the authors have extended He's homotopy perturbation method to solve second order strongly nonlinear generalized Duffing oscillators (He 1998, 1999, 2006, Younesian et al. 2010, Uddin et al. 2012) with small damping. It is too much difficult to solve the strongly nonlinear generalized Duffing type problems, especially with small damping and high order nonlinearities by the classical perturbation methods (Krylov 1947, Bogoliubov and Mitropolskii 1961, Mitropolskii 1964, Nayfeh 1981, Murdock 1991, Alam et al. 2006, Arya and Bojadziev 1981, Bojadziev 1983). But the suggested method has been successfully applied to solve second order strongly nonlinear generalized differential systems with small damping and high order nonlinearities. The first order approximate solutions of Eq. (13) and Eq. (25) are computed with small damping and high order nonlinearities by Eqs. (23) and (35), respectively and the corresponding numerical solutions are obtained by using fourth order Runge-Kutta method. The variational equations of the amplitude and phase variables are appeared in a set of first order ordinary differential equations. The integration of these variational equations is carried out by the well-known techniques of calculus (Nayfeh 1981, Murdock 1991). In a lack of analytical solutions, these are solved by numerical procedure (Lim and Wu 2003, Alam et al. 2006, Arya and Bojadziev 1981, Bojadziev 1983, Uddin et al. 2011, Uddin and Sattar 2011, Uddin 2011, Uddin et al. 2012). The amplitude and phase variables change slowly with time $t$. The behavior of amplitude and phase variables characterizes the oscillating processes and amplitude tends to zero in presence of small damping for large time $t$ (i.e., $t \rightarrow \infty$ ). On the other hand, the proposed technique can take full advantage of the classical perturbation methods. The solutions obtained by the presented method show a good agreement with those obtained by the numerical procedure with several damping effects. It is also noticed that the presented method is also capable to handle the second order weakly nonlinear generalized Duffing oscillators with damping effects and high order nonlinearities while the classical perturbation methods are unable to handle such situations. Comparisons are made between the solutions obtained by the presented technique and those obtained by the numerical procedure in Figs 1 - 2 for both strongly $(\varepsilon=1.0)$ and weakly $(\varepsilon=0.1)$ nonlinear generalized differential systems with small damping effects in graphically. Also the solution of the Duffing equation for cubic nonlinearity is obtained from Eq. (13) 
and Eq. (25) by setting $\alpha_{5}=0, \alpha_{7}=0$ with small damping (Figs 3 ) which agrees to the previous results (Uddin et al. 2011).

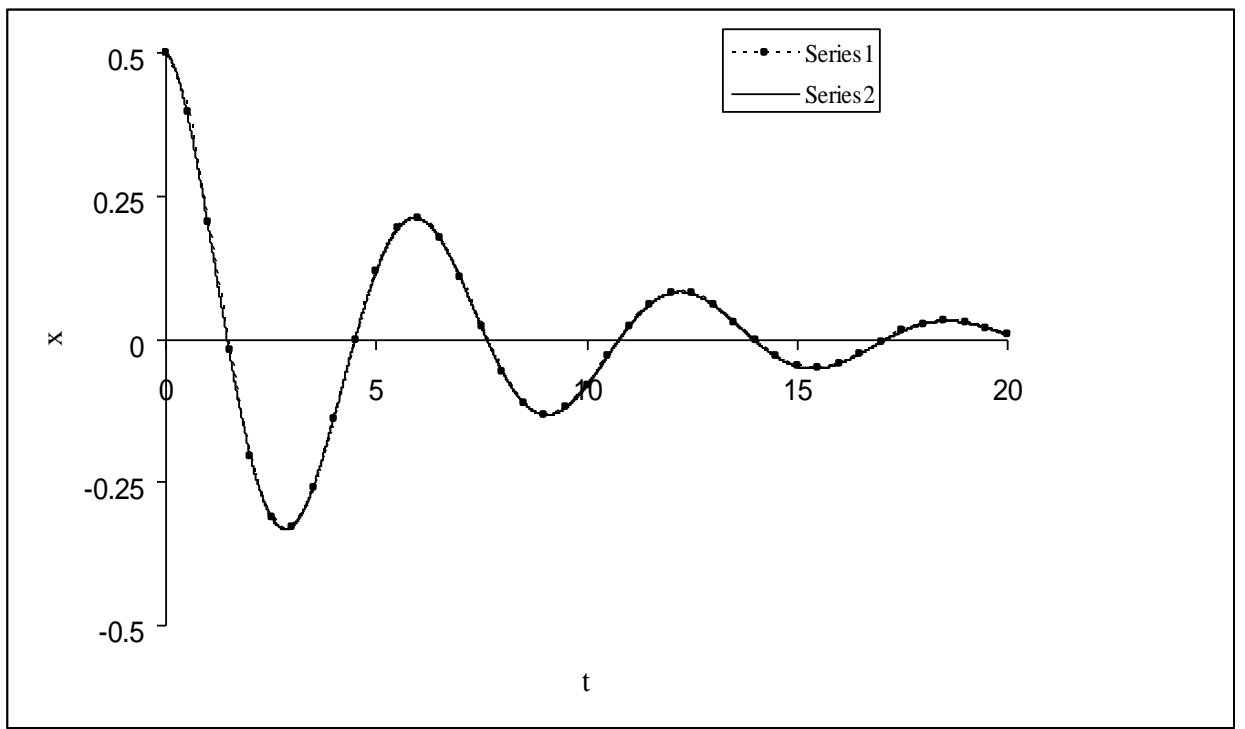

Fig. 1 (a). First approximate solution of Eq. (13) is denoted by -•- (dashed lines) by the presented analytical technique with the initial conditions $b_{0}=0.5, \Psi_{0}=0$ or $[x(0)=0.5, \dot{x}(0)=-0.07194]$ with $k=0.15, \varepsilon=1.0, \alpha_{3}=1.0, \alpha_{5}=1.0$ and $f_{3}=$ $x^{3}, f^{5}=x^{5}$. Corresponding numerical solution is denoted by - (solid line).

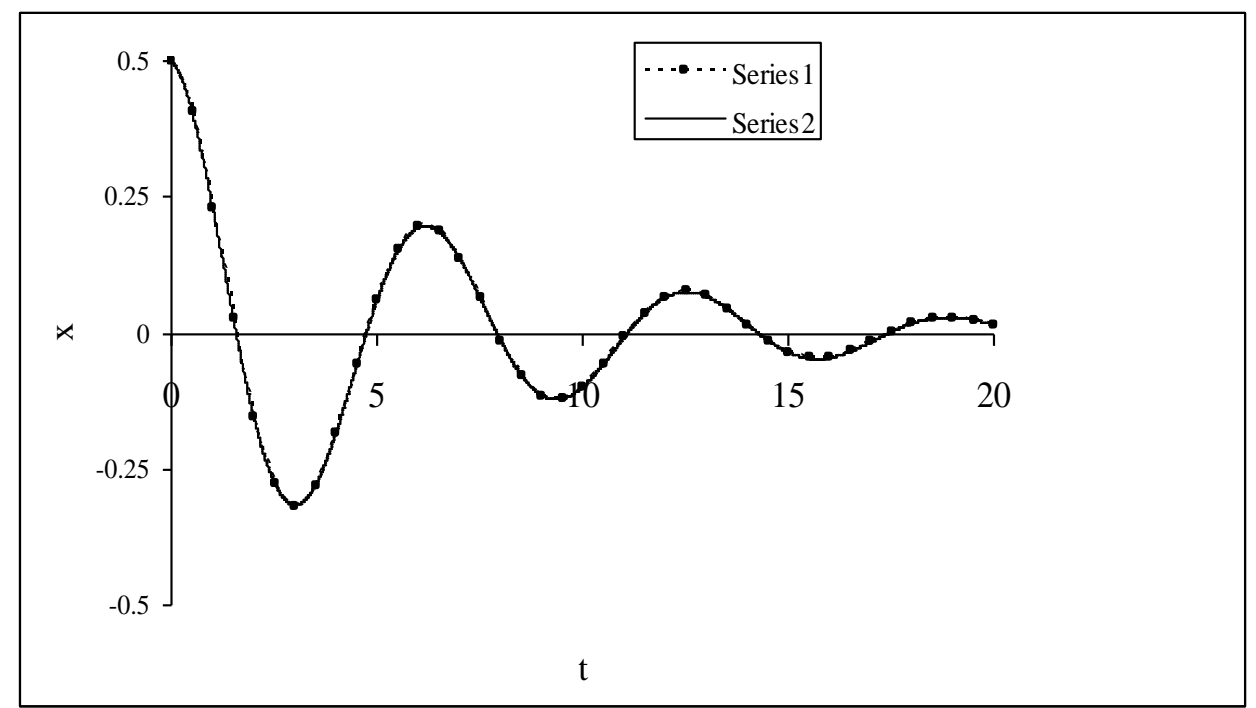

Fig. 1 (b). First approximate solution of Eq. (13) is denoted by -•- (dashed lines) by the presented analytical technique with the initial conditions $b_{0}=0.5, \Psi_{0}=0$ or $[x(0)$ $=0.5, \dot{x}(0)=-0.07466]$ with $k=0.15, \varepsilon=0.1, \alpha_{3}=1.0, \alpha_{5}=1.0$ and $f_{3}=x^{3}, f_{5}$ $=x^{5}$. Corresponding numerical solution is denoted by $-($ solid line $)$. 


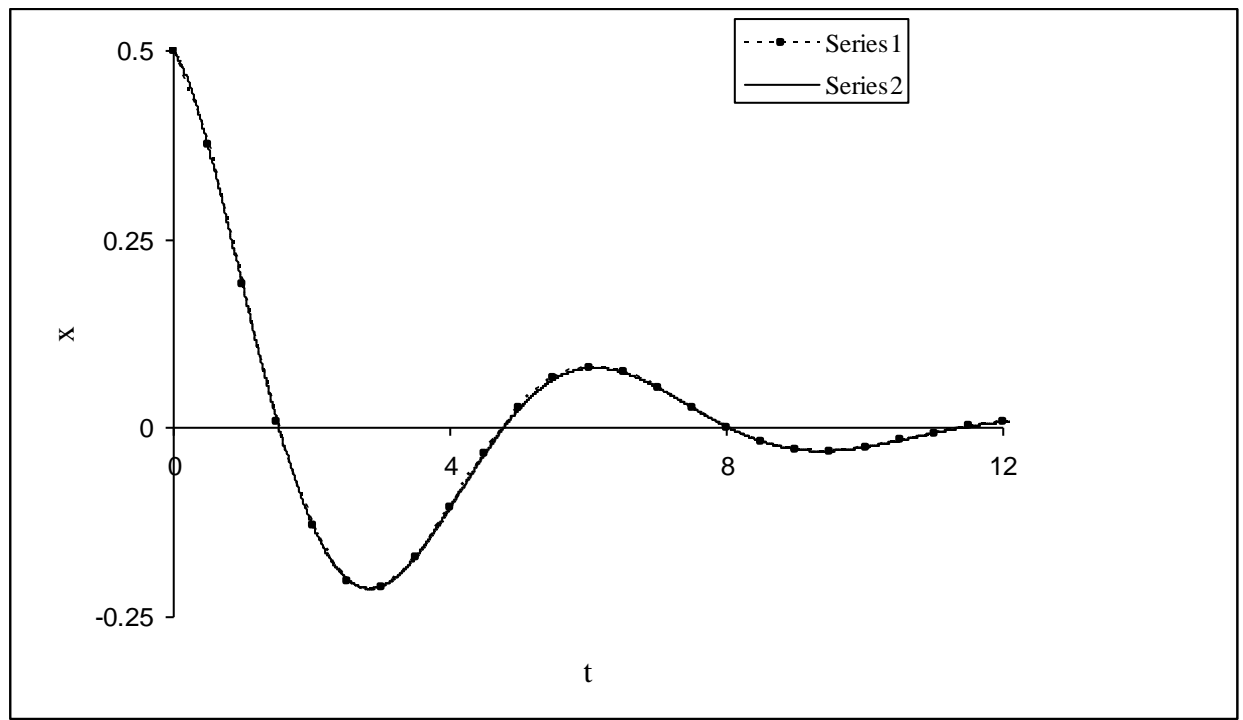

Fig. 2 (a). First approximate solution of Eq. (25) is denoted by $-\bullet-($ dashed lines) by the presented analytical technique with the initial conditions $b_{0}=0.5, \Psi_{0}=0$ or $[x(0)=0.5, \dot{x}(0)=-0.013401]$ with $k=0.3, \varepsilon=1.0, \alpha_{3}=1.0, \alpha_{5}=1.0, \alpha_{7}=$ 1.0 and $f_{3}=x^{3}, f_{5}=x^{5}, f_{7}=x^{7}$. Corresponding numerical solution is denoted by (solid line).

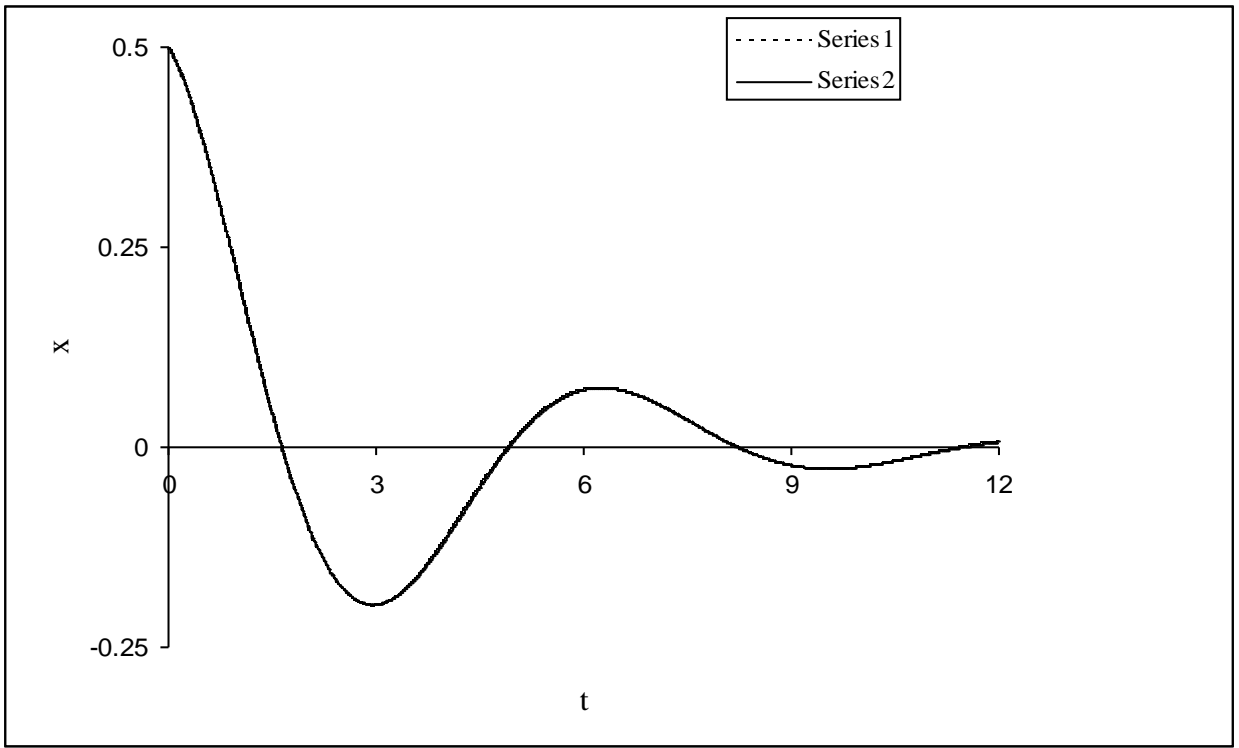

Fig. 2 (b). First approximate solution of Eq. (25) is denoted by - $\bullet-$ (dashed lines) by the presented analytical technique with the initial conditions $b_{0}=0.5, \Psi_{0}=0$ or $[x(0)$ $=0.5, \dot{x}(0)=-0.14781]$ with $k=0.3, \varepsilon=0.1, \alpha_{3}=1.0, \alpha_{5}=1.0, \alpha_{7}=1.0$ and $f_{3}$ $=x^{3}, f_{5}=x^{5}, f_{7}=x^{7}$. Corresponding numerical solution is denoted by - (solid line). 


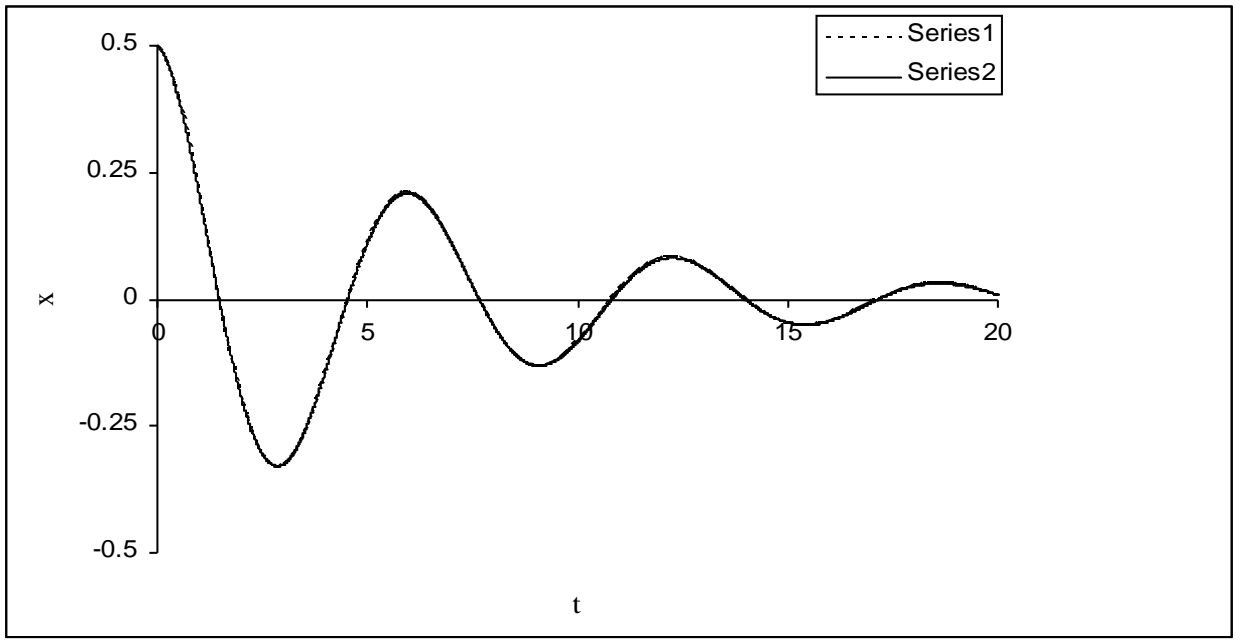

Fig. 3 (a). First approximate solution of Eq. (13) is denoted by --- (dashed lines) by the presented analytical technique with the initial conditions $b_{0}=0.5, \Psi_{0}=0$ or $[x(0)$ $=0.5, \dot{x}(0)=-0.07281]$ with $k=0.3, \varepsilon=1.0, \alpha_{3}=1.0, \alpha_{5}=0.0$, and $f_{3}=x^{3}$. Corresponding numerical solution is denoted by - (solid line).

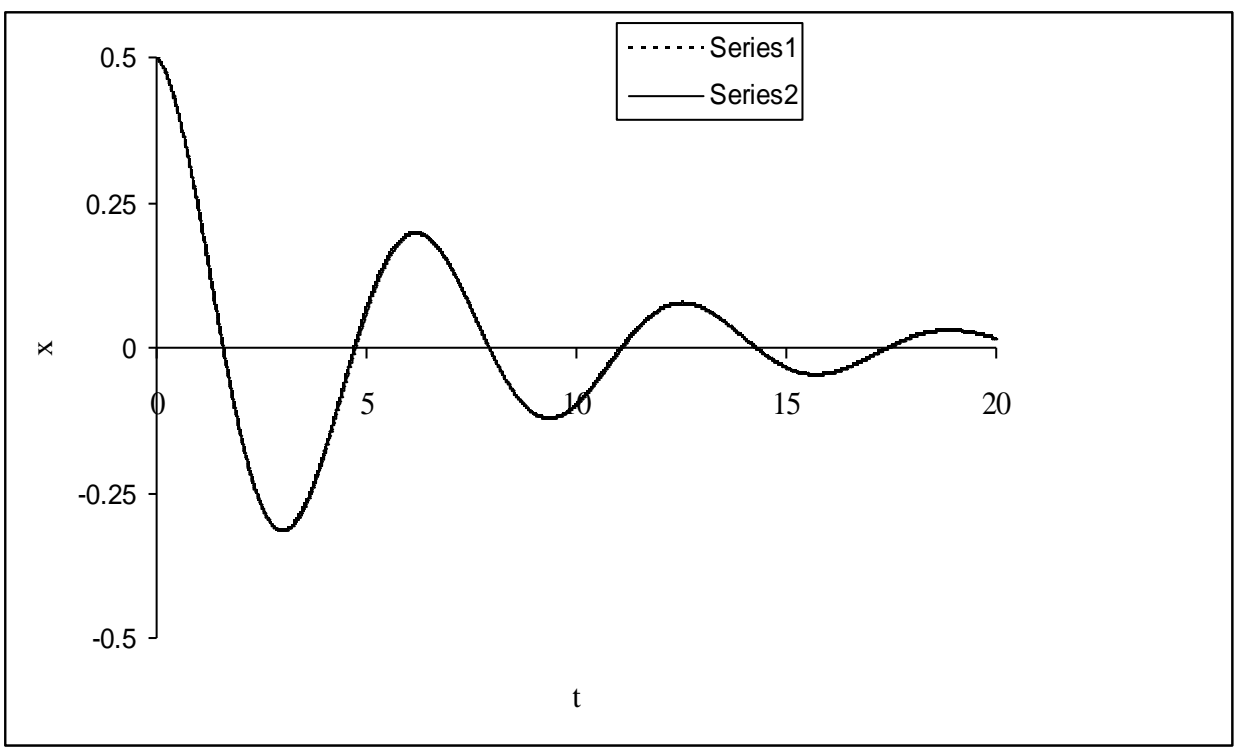

Fig. 3 (b). First approximate solution of Eq. (13) is denoted by --- (dashed lines) by the presented analytical technique with the initial conditions $b_{0}=0.5, \Psi_{0}=0$ or $[x(0)$ $=0.5, \dot{x}(0)=-0.07476]$ with $k=0.15, \varepsilon=0.1, \alpha_{3}=1.0, \alpha_{5}=0.0$, and $f_{3}=x^{3}$. Corresponding numerical solution is denoted by - (solid line).

\section{CONCLUSION}

The presented method has been successfully implemented to illustrate the effectiveness and convenience of the suggested procedure and it is noticed that the first 
order analytical approximate solutions show a good agreement with those obtained by the numerical procedure for second order strongly and weakly nonlinear generalized Duffing oscillator with damping while the classical perturbation methods are unable to handle such systems.

\section{REFERENCES}

Alam, M. S., M. A. K. Azad and M. A. Hoque. 2006. A general Struble's technique for solving an $n$th order weakly nonlinear differential system with damping. Int. J. Nonlinear Mechanics 41: 905-918.

Arya,J. C. and G. N. Bojadziev. 1981. Time depended oscillating systems with damping, slowly varying parameters and delay. Acta Mechanica 41: 109-119.

Azad, M. A. K., M. Samsuzzoha, M. A. Akbar and M. Alhaz. 2006. KBM asymptotic method for over-damped processes in biological and biochemical systems. Ganit: J. Bangladesh Mathematical Society 26: 1-10.

Bogoliubov, N. N. and Yu Mitropolskii. 1961. Asymptotic methods in the theory of nonlinear oscillation. Gordan and Breach, New York.

Belendez, A., A. Hernandez, T. Belendez, E. Fernandez, M. L. Alvarez and C. Neipp.2007. Application of He's homotopy perturbation method to Duffing harmonic oscillator. Int. J. Nonlinear Science and Numerical Simulation 8(1): 78-88.

Bojadziev, G. N. 1983. Damped nonlinear oscillations modeled by a 3-dimensional differential system. Acta Mechanica 48: 193-201.

He, J. H. 1998. Approximate solution of nonlinear differential equations with convolution product nonlinearities. J. Computer methods in applied mechanics and engineering 167: 69-73.

He, J. H. 1999. Some new approaches to Duffing equation with strongly and high order nonlinearity (I) linearized perturbation method. J. Communications in Nonlinear Science \& Numerical Simulation 4(1): 78-80.

He, J. H. 2006. New interpretation of homotopy perturbation method. Int. J. Modern Physics B: 20(18): 2561-2568.

Krylov, N.N. and N.N. Bogoliubov. 1947. Introduction to nonlinear mechanics. Princeton University Press, New Jersey.

Lim, C. W. and B. S. Wu. 2003. A new analytical approach to the Duffing- harmonic oscillator.J. Physics Letters A 311: 365-373.

Mitropolskii, Yu A. 1964. Problems on asymptotic methods of non-stationary oscillations (in Russian). Izdat, Nauka, Moscow.

Murdock, J. A. 1991. Perturbations: Theory and methods. Wiley, New York.

Nayfeh, A. H. 1981. Introduction to perturbation techniques. Wiley, New York.

Uddin M. A., M. A. Sattar and M. S. Alam. 2011. An approximate technique for solving strongly nonlinear differential systems with damping effects. Indian Journal of Mathematics 53(1): 83-98.

Uddin, M. A. and M. A. Sattar. 2011. An approximate technique to Duffing`equation with small damping and slowly varying coefficients. J. Mechanics of Continua and Mathematical Sciences 5(2): 627-642.

Uddin, M. A. 2011. He's homotopy perturbation method to Duffing equation with damping and high order (fifth and seven) strongly nonlinearities. Indian Journal of Theoretical Physics 59(3): 295-304. 
Uddin ,M. A. and M. A. Sattar. 2011. An approximate technique for solving strongly nonlinear biological systems with small damping effects. J. Calcutta Mathematical Society 7(1): 51-62.

Uddin, M. A., M. A. Alom and M. W. Ullah. 2012. An analytical approximate technique for solving a certain type of fourth order strongly nonlinear oscillatory differential system with small damping. Far East Journal of Mathematical Sciences 67(1): 59-72.

Younesian, D., H. Askari, Z. Saadatnia and M. K. Yazdi. 2010. Frequency analysis of strongly nonlinear generalized Duffing oscillators using He's frequency- amplitude formulation and He's energy balance method. J. Computers and Mathematics with Applications 59: $3222-$ 3228 .

(Received revised manuscript on 13 April, 2015) 